\title{
Stepwise Investment Plan Optimization for Large Scale and Multi-Zonal Transmission System Expansion
}

\author{
Hakan Ergun Graduate Student Member, IEEE, Barry Rawn Member, IEEE, \\ Ronnie Belmans Fellow, IEEE and Dirk Van Hertem Senior Member, IEEE
}

\begin{abstract}
This paper develops a long term transmission expansion optimization methodology taking the probabilistic nature of generation and demand, spatial aspects of transmission investments and different technologies into account. The developed methodology delivers a stepwise investment plan to achieve the optimal grid expansion for additional transmission capacity between different zones. In this paper, the optimization methodology is applied to the Spanish and French transmission systems for long term optimization of investments in interconnection capacity.
\end{abstract}

Index Terms-Transmission System Expansion Planning, Transmission System Optimization, Optimal Cable Routing, HVDC, Transmission System Investments, Time Point Optimization

\section{INTRODUCTION}

The European electricity sector is currently facing major changes and challenges. Due to the European 20-20-20 targets, the share of generation from renewable energy sources in the total generation keeps increasing steadily. This has a major impact on the generation mix and the way the transmission grid is operated. By the end of this decade, the total installed capacity of wind power plants is expected to reach $18.9 \%$ of the total installed generation capacity in Europe [1]. Including solar generation, hydro generation and other renewable sources, the total share of installed generation capacity from renewable energy sources will reach $54.4 \%$ by 2020 [1]. In 2014, $79.1 \%$ of new installed generation capacity used renewable energy sources [2].

The increased power flows and their volatility in the European transmission network makes significant investments inevitable. ENTSO-E estimates that $52300 \mathrm{~km}$ of new or refurbished extra high voltage routes will be required in the coming decade, equal to an investment volume of 104 billion $€[3]$. As transmission grid planning over long time horizons is subjected to a large number of uncertainties, robust optimization methodologies need to be developed, allowing for the analysis of many different scenarios and help to minimize investment risks. At the same time, these methods must be computationally feasible.

This work is supported by ABB, Elia and Vattenfall in the framework of the industrial research project "Reaching for a Pan-European Grid: How to Make the Transition? - Transmission System Investment Planning".

H. Ergun, R. Belmans and D. Van Hertem are with the Department of Electrical Engineering (ESAT/ELECTA), KU Leuven being a founding member of EnergyVille, Kasteelpark Arenberg 10, 3001 Heverlee, Belgium, http://www.esat.kuleuven.be/electa.

B. Rawn is with Deptarment of Electronic and Computer Engineering, Brunel University, London, United Kingdom

Contact: hakan.ergun@esat.kuleuven.be
In general, transmission system investment optimization is a difficult task due to the non-linear, non-convex and mixed-integer nature of the optimization problem [4]. The liberalization of the electricity market and increased use of energy from renewable energy sources increase the level of complexity in determining the optimal location and size of new investments. In the literature, a large number of transmission system optimization methodologies exist, as extensively outlined in [4] and [5]. These methodologies can be classified in several ways as proposed in [4].

The first possible classification is the formulation of the optimization problem [4]. Mathematical optimization methods include linear programming [6]-[8], quadratic programming, non-linear programming [9], integer programming and mixedinteger programming [10]-[14] and their combinations. For high dimensional non-convex problems these methods become computationally expensive and have been enhanced by heuristic methods [15]-[17]. The most popular include sensitivity analysis [18], [19], genetic algorithms [20]-[25], simulated annealing [26]-[28], tabu search [29], [30] and particle swarm optimization [31], [32].

Another possible classification is the time horizon of the used methodology. Static optimization methodologies deliver only the solution for a specific time point of the planning horizon, whereas dynamic tools provide a sequence of optimal investments over the entire time horizon [4]. The most popular approaches for dynamic optimization methodologies use dynamic programming [33]-[35] and several heuristic approaches [11], [28], [36]-[41]. Nevertheless, dynamic planning methodologies including multiple time steps are still not sufficiently developed and need often major simplifications [42].

The assumed structure of the power system is a third possibility to classify transmission expansion optimization methodologies. The majority of the reviewed literature describes methodologies where the planning is carried out by a single entity. In case several planning entities are simulated, mostly game theory based methods are used [43]-[46].

Above mentioned types of transmission system expansion optimization methodologies can be defined in a deterministic way as well as using probabilistic approaches [47][50]. Depending on the used solution methodology, planning horizon and power system structure, still major simplifications have to be made in order to solve this complex optimization problem. Most successful optimization methodologies achieve scalability by decomposing the problem at hand into different layers [7], [51], [52]. By using decomposition methods the 
optimization problem divided into several virtually decoupled layers. The different optimization problems are solved in a more simplified and efficient way. Eventually, these layers have to interact with each other, exchange dedicated information and solve the expansion problem iteratively. [53] provides different decomposition methods and several applications of these for power system optimization including transmission expansion planning.

A key short-coming of available planning methodologies is that spatial aspects of transmission assets and the use of different technology options for new investments are not taken into account, especially in dynamic planning methodologies. This work provides a methodology to determine optimal time points, rating, location, routing and technology of new transmission investments in a given area, taking into account spatial aspects. As a result, a detailed and optimized stepwise investment plan for a defined planning horizon is obtained. The developed methodology can be used by transmission system operators to efficiently analyse different future scenarios in order to reduce risk for future investments. This work extends the static model presented in [54] to a pseudo-dynamic model. The use of the $\mathrm{A}^{*}$ shortest path algorithm has been introduced to improve the computational efficiency and enable practical applications. The formulation presented makes it possible to assess the effects of delays in corridor availability, availability of transmission technology and constraints on investments.

The paper is is organized as follows. Section II illustrates the structure of the proposed long term planning methodology. Section III describes the optimization of network topology and investment time points. Section IV explains how the optimization of transmission routes and technologies is performed. In Section V a simple case study is provided to illustrate the working principle of the developed algorithm. Section VI shows the application of the methodology to a larger case study. Finally, in Section VII conclusions are drawn.

\section{Proposed LONG TERM PLANNING METHOdOLOGY AND OPTIMIZATION METHODOLOGY}

The methodology illustrated in this paper uses two iterative optimization layers consisting of dynamic optimization of investments and an optimization of used technology and transmission routes considering spatial aspects. Fig. 1 shows the structure of the proposed long term transmission expansion optimization methodology. The different building blocks of the methodology are discussed briefly in the following paragraphs. The main focus of this paper is on the third block, the substation level.

\section{A. Interconnection level}

The interconnection level determines the capacity needs between multiple zones, considering future generation and demand scenarios to enable long term security of supply. The objective of the optimization is to find the best interconnection capability between multiple zones maximizing social welfare. The output of the interconnection level is the optimal interconnection capacity and generation cost information. The determination of the interconnection level is not in the scope

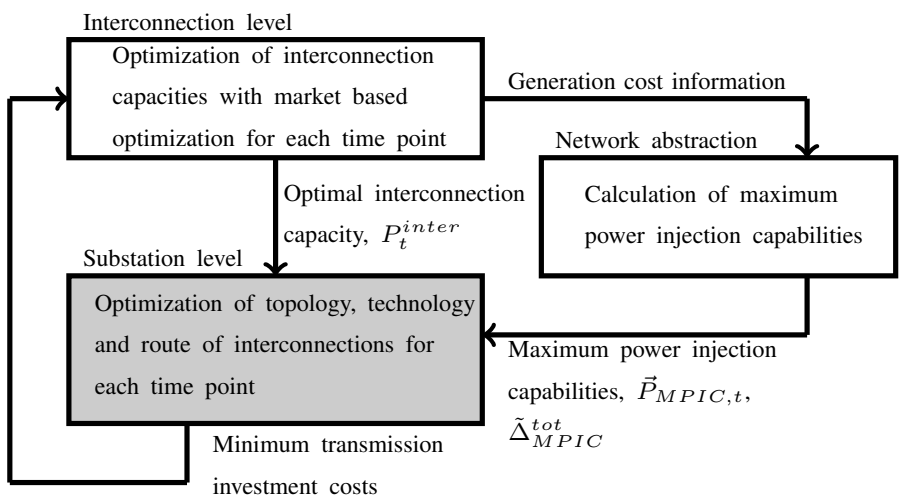

Fig. 1: Structure of the proposed long term transmission expansion optimization methodology

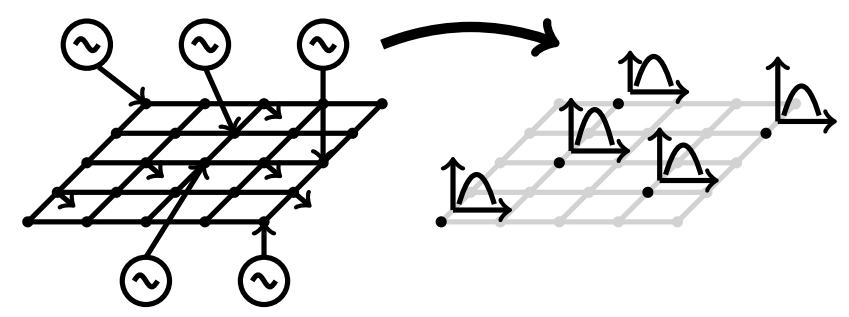

Existing grid

Abstract Network

Fig. 2: Principle of network abstraction [56]

of this work as a large number of such market based tools exist [55].

\section{B. Network Abstraction}

Using the input coming from the interconnection level, an optimization algorithm can be written to determine optimal location and rating of new assets to achieve the desired interconnection power. If the existing transmission system is modelled with its full detail and technology selection, while route and timing of the investments are optimized, the number of variables becomes a burden. Therefore, an abstraction from the existing network is created to decouple the optimization of technology, topology, routing and timing of new investments from the existing network.

The principle of the network abstraction is shown in Fig. 2. In the abstract layer, the existing network is presented as a possible set of allowable injections and absorptions. The allowable injections and absorptions are determined considering probabilistic distribution of generation and demand as well as existing line limits. The determined injection capabilities indicate the maximum amount of power which can be imported or exported in a particular node of the system without causing any overload situations in the existing network. By doing so, a set of selected nodes can be used for the further optimization at substation level, rather than the entire set of nodes. The calculation methodology for the network abstraction is briefly illustrated in Appendix A of this paper. More detailed information can be found in [54], [56], [57]. 


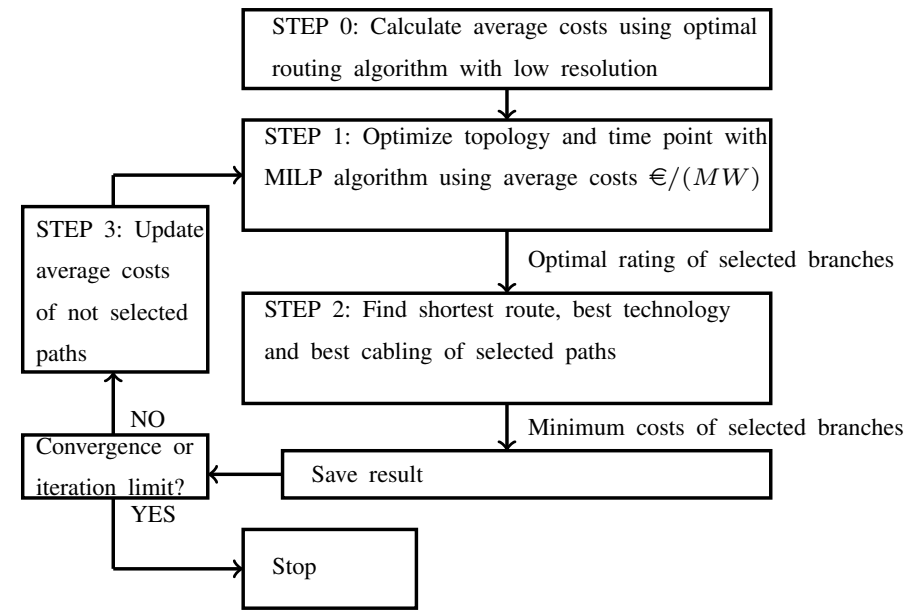

Fig. 3: Iterative optimization of transmission topology, technology, cabling, routing and time point

\section{Substation level}

At the substation level, which is described in the remainder of this paper, the optimization of new investments is carried out. The optimization determines for each time point of the planning horizon which substations to connect, which technology (AC, DC, overhead lines, underground cables) and power rating to use such that the desired interconnection capability is achieved in the economically most feasible way.

The optimization considers area dependent installation costs and finds the cheapest transmission route in combination with the technology. This way, soft constraints such as social and environmental impact can be included in these area dependent costs as illustrated in Section VI. Using different constraints, the impact of construction delays and availability of multiterminal HVDC operation is analysed.

Fig. 3 shows the structure of the investment optimization. The investment optimization uses iteratively an MILP optimization and a shortest path algorithm. In the initial step, average transmission system costs are calculated. The costs are calculated using the optimal routing algorithm described in Section IV with a low spatial resolution only for all possible branches and a few chosen power ratings (STEP 0, Fig. 3). The average costs for other all power ratings are linearly approximated. Using these costs as input, a mixed integer linear problem (MILP) is solved where the topology (which substations/nodes are connected with each other), power rating and time point of new grid investments are determined (STEP 1, Fig. 3). The objective of the optimization is the minimization of total transmission system costs over the planning horizon. The problem formulation is provided in Section III.

Using the provided topology and power ratings, the transmission technologies and routes of each selected branch are optimized, taking into account area dependent installation costs (STEP 2, Fig. 3). The objective is the minimization of the costs separately for each selected branch. This procedure is based on an optimal routing algorithm which is explained in Section IV.

The original selection of ratings must be revisited in the next iteration. Therefore the average costs are updated (STEP
3) with the minimum costs obtained by the optimal routing algorithm (STEP 2) and fed into the MILP optimisation (STEP 1). In order to avoid that the algorithm gets stuck in a local optimum, penalty functions are used for branches and power ratings which have not been selected. The following penalty functions are used

$$
c_{N S}^{z+1}= \begin{cases}c_{N S}^{z} \cdot\left(1-\overline{c_{d i f f}}\right)^{(3 / 4)} & \text { if } \overline{c_{\text {diff }}} \geq 0 \\ c_{N S}^{z} \cdot\left(1+\overline{c_{\text {diff }}}\right)^{(3 / 4)} & \text { if } \overline{c_{\text {diff }}}<0\end{cases}
$$

where $c_{N S}^{z}$ and $c_{N S}^{z+1}$ are the costs of not selected branches in iterations $z$ and $z+1$ respectively, and $\overline{c_{\text {diff }}}$ is the average of the relative cost differences between iterations $z$ and $z-1$ for the branches selected in iteration $z$. The steps 1 to 3 are repeated until the relative cost difference $c_{\text {diff }}$ between two iterations is below a certain threshold $\epsilon$ or a maximum number of iterations have been reached. For the calculations shown in this paper, $\epsilon$ is defined as $10^{-5}$.

\section{OPTIMIZATION OF INVESTMENT TIME POINTS AND GRID TOPOLOGY}

This section shows how the optimization of the grid topology and investment time points delivering a stepwise investment plan are carried out using mixed-integer linear programming (MILP). Equation (III) shows the objective function of the MILP problem statement. The objective of the optimization problem is the minimization of transmission system costs over the entire planning horizon consisting of investment and installation costs. The search space consists of $N_{s}=2 \cdot N_{b} \cdot N_{k} \cdot N_{t}$ binary decision variables, where $N_{b}$ is the number of possible branches, $N_{k}$ the number of possible power ratings and $N_{t}$ is the number of investment time points. $U_{i j t}$ is a binary decision variable defined for each possible branch $i$, power rating $j$ and time point $t$. As each branch can carry power flows in both directions, two decision variables $U_{i j t}^{+}$and $U_{i j t}^{-}$are used to account for different power flow directions.

$$
\begin{aligned}
\min _{\substack{\forall U^{+}, U^{-} \\
\in B^{N_{b} \times N_{k} \times N_{t}}}} C_{c a p} & =\left(U^{+}+U^{-}\right):(\overrightarrow{1} \otimes \mathbf{k}):(\overrightarrow{1} \otimes \mathbf{t}) \cdot \tilde{C} \\
& =\sum_{i} \sum_{j} \sum_{t}\left(U_{i j t}^{+}+U_{i j t}^{-}\right) \cdot K_{i j t} \cdot \tilde{C}_{i j t}
\end{aligned}
$$

Vector $\mathbf{k}$ contains all possible power ratings of transmission paths. Vector $\tilde{C}$ contains costs for each transmission path, power rating and investment time point. For instance $C_{1,2,3}$ is the investment costs of path 1 , using power rating 2 , at investment time point 3 . The elements of $\tilde{C}$ are defined separately for each optimization time point $t$. As future expenses are worth less in today's currency, the elements of $\tilde{C}$ are depreciated over time using (3) and (4), where $q$ is a user defined discount rate.

$$
\begin{aligned}
\tilde{C} & =\left[C_{i j, t=0}, C_{i j, t=1}, \ldots, C_{i j, t=N_{t}}\right] \\
C_{i j, t=x} & =C_{i j, t=0} \cdot(1-q)^{x}, \quad 1 \geq q \geq 0
\end{aligned}
$$


As the interest rate is greater than zero, future costs are always lower than the costs at the starting year of the planning horizon. Thus, the optimization solver postpones more expensive investments as long as possible, as they have a higher depreciation. The investments can only be postponed as far as the optimization constraints, which are shown (5) - (10), allow it.

$$
\begin{aligned}
P_{t}^{\text {inter }} & \leq\left(U^{+}+U^{-}\right): \mathbf{l} \otimes \mathbf{k}, \quad \forall t \in\left[1, N_{t}\right] \\
P_{M P I C, t} & \geq \tilde{\Delta}_{M P I C}^{t o t} \cdot\left(U^{+}+U^{-}\right) \cdot \mathbf{k}, \quad \forall t \in\left[1, N_{t}\right] \\
\overline{\mathbf{C}}_{\mathbf{t}} & \geq\left(U^{+}+U^{-}\right):(\overrightarrow{1} \otimes \mathbf{k}) \cdot \tilde{C} \quad \forall t \in\left[1, N_{t}\right] \\
0 & =U_{d l}^{+}+U_{d l}^{-} \forall t \leq \mathbf{t}_{\mathbf{d l}}, \\
\overrightarrow{1} & \geq E_{h v d c} \cdot\left(U_{h v d c}^{+}+U_{h v d c}^{-}\right) \quad \forall t \leq t_{H V D C} \\
K_{i} & \leq \bar{K}, \forall i \in\left[1, N_{b}\right]
\end{aligned}
$$

Constraint (5) states that the sum of power ratings of tie lines between zones must be greater or equal than the desired interconnection power $P_{t}^{\text {inter }}$ for each time step of the planning horizon. $\mathbf{l}$ is a vector indicating tie lines between the considered zones as defined in (11) and $\mathbf{k}$ is the vector of possible power ratings.

$$
\mathbf{l}=\left\{\begin{array}{cc}
1 & \text { if branch } i \text { is a tie line } \\
0 & \text { otherwise }
\end{array}\right.
$$

Constraint (6) states that the maximum power injection capabilities of candidate nodes may not be exceeded. $\tilde{\Delta}_{M P I C}^{t o t}$ is defined as a $\left(N_{N} \cdot N_{t}\right) \times\left(N_{b} \cdot N_{k} \cdot N_{t}\right)$ matrix, $N_{N}$ being the number of candidate nodes. The elements of $\tilde{\Delta}_{M P I C}^{t o t}$ contain the matrices $\Delta_{M P I C}^{t o t}$ which indicate the change in the injection capabilities of candidate nodes depending on the power ratings of possible branches at each time point (12). The size of $\Delta_{M P I C}^{t o t}$ is $N_{N} \times\left(N_{b} \cdot N_{k}\right)$. The binary decision variables $U$ are defined separately for each time point and a specific branch can only be selected at one specific time point. By defining $\tilde{\Delta}_{M P I C}^{t o t}$ as given in (12), the selected branches of each time point influence the maximum power injection capabilities from the time point they have been selected to the end of the planning horizon. Hence, their presence to the end of the planning horizon is ensured using constraint (6). A brief description of the calculation methodology for $\Delta_{M P I C}^{t o t}$ has been provided in Appendix A. Detailed information about the calculation can be found in [54], [56], [57].

$$
\tilde{\Delta}_{M P I C}^{t o t}=\left[\begin{array}{cccc}
\Delta_{M P I C, t=0}^{t o t} & 0 & \cdots & 0 \\
\Delta_{M P I C, t=1}^{t o t} & \Delta_{M P I C, t=1}^{t o t} & \cdots & 0 \\
\vdots & \vdots & \vdots & \vdots \\
\Delta_{M P I C, t=N_{t}}^{t o t} & \Delta_{M P I C, t=N_{t}}^{t o t} & \cdots & \Delta_{M P I C, t=N_{t}}^{t o t}
\end{array}\right]
$$

Usually, transmission system operators have to find the necessary capital for transmission system investments on the capital market. The amount of capital made available for transmission system operators is determined by their financial status and is limited for a certain investment period. Constraint (7) takes the limitation of capital availability into account and states that the sum of investments in a particular time step may not be higher than a defined maximum investment $\bar{C}_{t}$. The $1 \times N_{t}$ vector $\overline{\mathbf{C}}_{\mathbf{t}}$ contains the maximum investments per term which can have different values at each investment time point as shown in (13). By varying the elements of $\overline{\mathbf{C}}_{\mathbf{t}}$, several different investment sequences and grid configurations can be obtained. This helps the grid planner to analyse the effects of capital availability on the final grid configuration and on the total net present value of investment sequences in order to prioritize investment decisions and reduce investment risks.

$$
\overline{\mathbf{C}}_{\mathrm{t}}=\left[\overline{\mathbf{C}}_{1}, \overline{\mathbf{C}}_{2}, \ldots, \overline{\mathbf{C}}_{\mathrm{N}_{\mathrm{t}}}\right]
$$

Transmission system investment projects face very often delays due to internal and external factors. Internal factors include delays in the supply chain for equipment as well as delays in construction due to unforeseen technical or geographical obstacles. External factors include delays in the planning and permission process, mostly due to public resistance, policy or financial issues. It is important to analyse the effect of possible delays on the final transmission grid topology and the resulting costs in order to identify priority corridors. Constraint (8) includes possible delays in the optimization model. The constraint states that certain transmission paths can only be built after a pre-defined time point of the planning horizon. $U_{d l}^{+}$and $U_{d l}^{-}$are a sub-set of the binary decision variables $U^{+}$ and $U^{-}$respectively, defining transmission paths facing delays. The vector $\mathbf{t}_{\mathbf{d l}}$ assigns time points to each element of the subsets $U_{d l}^{+}$and $U_{d l}^{-}$from where on connections on these paths may be established. For instance, to determine how long the construction of a line can be postponed, the time point in the delay vector can be increased step by step, until no feasible solution is found or the connection becomes obsolete in the final grid topology. This way a prioritization of different paths can be determined and justified.

Another interesting aspect in long term planning is technology availability. Depending on technology availability, the final grid topology and the final investment costs can be different. Constraint (9) has been introduced in the problem formulation to analyse the effect of availability of meshed HVDC configurations. The constraint states that for all time points $t$ which are smaller than a defined time point $t_{H V D C}$ no multi-terminal or meshed HVDC configuration is possible. $U_{h v d c}^{+}$and $U_{h v d c}^{-}$indicate the decision variables of paths which are built in HVDC and $E_{h v d c}$ is an incidence matrix. $E_{h v d c}$ has the size $N_{N} \times N_{b}$, where $N_{N}$ is the number of candidate nodes and $N_{b}$ is the number of possible branches. For instance, the elements $E_{m, i}$ and $E_{n, i}$ of $E_{H V D C}$ are 1 if branch $i$ is an element of $U_{h v d c}^{+}$and $U_{h v d c}^{-}$and connects nodes $m$ and $n$. All other elements are zero. This way, only one HVDC connection can be connected to one single node. This constraint is relaxed after the defined time period $t_{H V D C}$ such that more HVDC connections can be established the nodes $n$ and $m$. Similar to the line delay constraint, the variation of $t_{H V D C}$ can be used to investigate until when the technological maturity of multi-terminal HVDC configurations must be reached so it is still economically feasible to build such configurations.

Constraint (10) states that the maximum power rating of a connection path may not exceed a pre-defined maximum rating $\bar{K}$. 


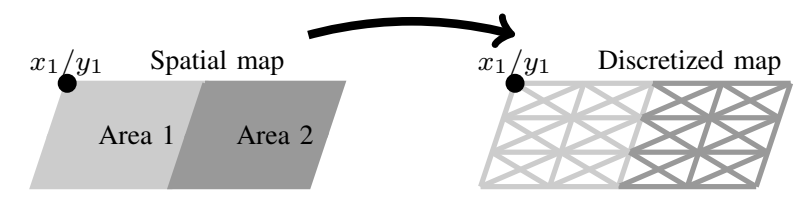

Fig. 4: Creation of a weighted directed graph by discretizing a spatial map

\section{OPTIMIZATION OF TECHNOLOGY AND ROUTING}

In transmission investment planning, it is not sufficient to consider only overhead lines for this purpose. Due to increased public opposition, permissions to build overhead lines (OHL) are hard to obtain [58]. When it comes to AC underground cables (UGC), the possible transmission distance is limited due to the high cable capacitance and the resulting charging currents. Therefore, the possibility of using HVDC transmission has to be considered during the optimization process.

The route and transmission technology are interdependent. Additionally, the installation costs for different technologies depend on the type of soil, land acquisition costs and other factors. It is therefore important to optimize the cabling option (OHL vs. UGC), technology (HVAC vs. HVDC) and the transmission route at the same time. In the developed algorithm, a map of the considered installation area containing all candidate nodes is discretized to a grid of spatial points $p_{i}=\left[x_{i}, y_{i}\right]$ of size $N_{x} \times N_{y}$ as shown in Fig. 4. The number of chosen nodes and their horizontal and vertical position depend on the desired spatial resolution and the size of the area. All points $p_{i}=\left[x_{i}, y_{i}\right]$ are distributed in equal distance from each other.

To be able to optimize the technology together with the cabling option, the four technologies of AC OHL, AC UGC, DC OHL and DC UGC must also be represented, as well as the effect of creating hybrid options such as AC with DC and OHL with UGC. Therefore, the optimal routing and technology problem is formulated around a weighted graph $\mathcal{G}=(\mathcal{V}, \mathcal{E})$ having four technology layers and weighted edges signifying costs. This means that for each technology option a separate discretization is performed. For every spatial point $p$, there are four associated vertices $\mathcal{V}$ representing possible technologies. A full set of edges $\mathcal{E}$ between vertices associated with adjacent spatial points correspond to cables or lines of a given technology. There are also edges between the vertices associated with different technologies at the same spatial point $p$ that correspond to mechanical or electrical conversions necessary to join dissimilar technologies. This means that for a graph with $N_{x} \cdot N_{y}$ nodes, $\left(10 \cdot N_{x} \cdot N_{y}-3 \cdot\left(N_{x}+N_{y}\right)+2\right)$ edges exist.

The graph cost function $\mathcal{W}\left(p_{i}, \mathcal{E}_{j}\right)$ can take on a different positive value for every edge $j$ and represents the cost associated with progressing spatially or switching between technologies:

$$
\mathcal{W}\left(p_{i}, \mathcal{E}_{j}\right)=\left(c^{i n v}\left(p_{i}, \mathcal{E}_{j}\right)+c^{i n s t}\left(\mathcal{E}_{j}\right) \cdot w\left(p_{i}\right)\right) d\left(\mathcal{E}_{j}\right)+w^{\text {switch }}\left(\mathcal{E}_{j}\right) \quad(14)
$$

where $c^{i n v}\left(p_{i}, \mathcal{E}_{j}\right)$ and $c^{i n s t}\left(\mathcal{E}_{j}\right)$ correspond to both location and technology dependent investment and installation costs, respectively. $w\left(p_{i}\right)$ is a spatial weighting factor to take the

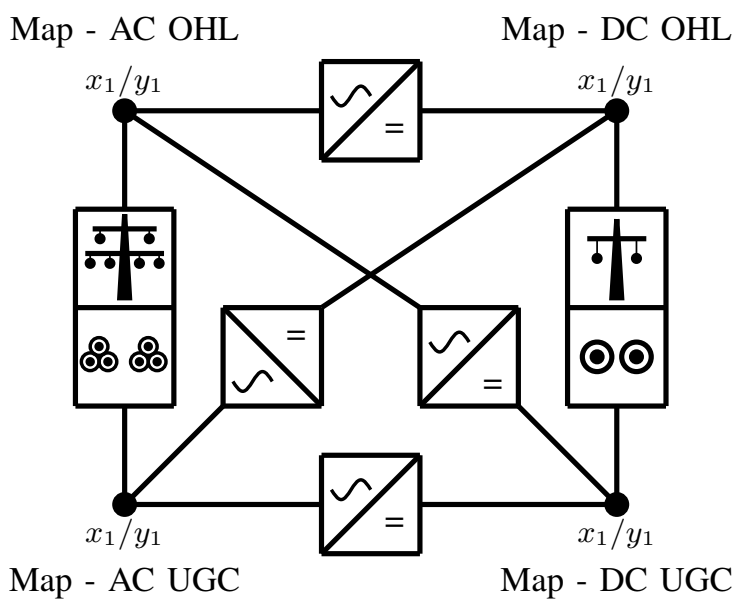

Fig. 5: Creation of a weighted directed graph containing four technologies as input for the shortest path algorithm [54]

change of installation costs in different areas into account. $d\left(\mathcal{E}_{j}\right)$ is the spatial distance associated with the edge, and $w^{\text {switch }}\left(\mathcal{E}_{j}\right)$ is a technology conversion cost. The cost factors are also dependent on the optimal capacity $K$ determined by the other sub-problem, but this is omitted as the quantity is fixed for a given iteration.

In (14), $c^{i n v}\left(p_{i}, \mathcal{E}_{j}\right)$ and $c^{i n s t}\left(\mathcal{E}_{j}\right)$ are given in $€ / k m$ for a defined reference area. The spatial weight $w\left(p_{i}\right)$ defines the relative difference of installation costs in a certain area compared to the reference area.

The weighting factor $w^{\text {switch }}\left(\mathcal{E}_{j}\right)$ is assigned to each edge of the graph to represent costs of technology switch in the weighted graph. Fig. 5 shows how the technology switch is realized. The four technology maps are connected at each vertex $x_{i} / y_{i}$ to each other. In case of a switch from AC to DC or DC to AC, the edge is weighted with the cost of an HVDC converter. In case of a switch within the same technology to a different cabling option, the weight is determined using the necessary number of cable systems and conductors per cable system (Fig. 5). If there is no switch in technology or cabling option, $w_{i}^{\text {switch }}$ is zero.

Including the above mentioned costs into a weighted graph, the shortest distance between the starting point and the termination point of a path can be determined using shortest path algorithms. If the weights assigned to the edges of the graph are costs per $\mathrm{km}$ of transmission line, the shortest path algorithm delivers the minimum transmission system cost between two vertices (15).

As all four technology layers are interconnected, the shortest path algorithm delivers the minimum transmission system cost

$$
\min _{S} C_{\text {equip }}=\sum_{\mathcal{E} \in \mathcal{S}} \mathcal{W}(\mathcal{E})
$$

where $S$ is a sequence of edges corresponding to the lowest cost or "shortest" path. Although Dijkstra's shortest path algorithm [59] has been used in previous work [54], in this work the heuristic $A^{*}$ algorithm has been implemented which has proven to be computationally more efficient [60]. The $\mathrm{A}^{*}$ algorithm is described in the Appendix B. 


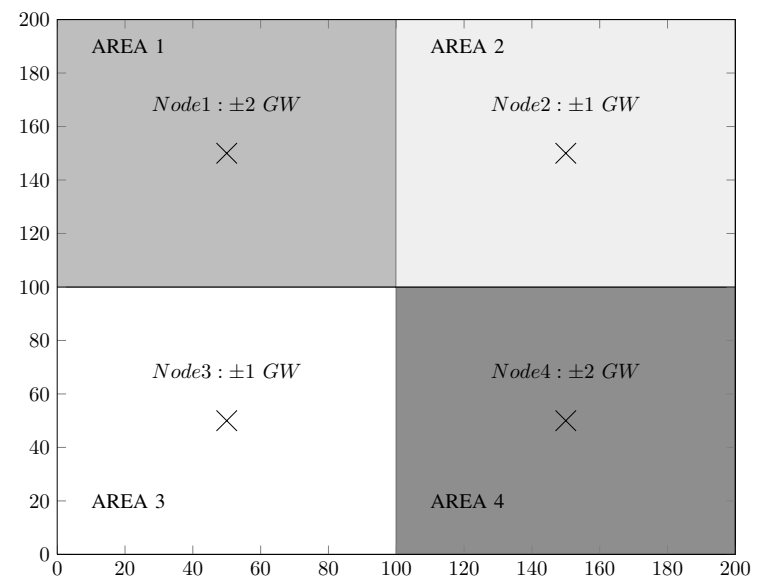

Fig. 6: Simple case study to demonstrate working principle

\section{CONCEPT CASE}

In this section, the developed methodology is applied to a very simple case to demonstrate the working principle. The case study consists of four nodes and four different areas as shown in Fig. 6. Nodes 1 and 2 belong to the same transmission zone, whereas nodes 3 and 4 form another transmission zone. It is assumed that in node $1,2 \mathrm{GW}$ can be injected and absorbed. Nodes 2, 3 and 4 can inject and absorb $1 \mathrm{GW}, 1 \mathrm{GW}$ and $2 \mathrm{GW}$ respectively. For the sake of simplicity it is assumed that the injection and absorption capabilities do not change which means that $\tilde{\Delta}_{M P I C}^{t o t}=0$.

Two different technology options have been used in this simple case having the same investment costs but different installation costs in each area. The investment costs are calculated using

$$
C_{i n v}=P / 1500+1 ;
$$

where $C_{i n v}$ is given in $€ / \mathrm{km}$ and $P$ is the power rating $M W$. For a $1500 \mathrm{MW}$ link, the costs would equal to $2 € / \mathrm{km}$. The basic installation cost is assumed to be $1 € / \mathrm{km}$. This means that the total costs of both links would be $3 € / \mathrm{km}$ in the base installation area. The specific weights for the installation costs and the resulting costs per km for a $1000 \mathrm{MW}, 1500 \mathrm{MW}$ and $2000 \mathrm{MW}$ are given in Tab. I. For the sake of simplicity, it is assumed that there is no additional cost of technology switch. During the optimization, the power rating of each path can be multiples of $100 \mathrm{MW}$. It is assumed that the maximum power rating of one path, $\bar{K}$, is $3 \mathrm{GW}$.

\section{TABLE I}

\section{SPECIFIC WEIGHTS FOR INSTALLATION COSTS}

\begin{tabular}{ll|cccc} 
Technology & Area & $\begin{array}{c}\text { Specific } \\
\text { weight }\end{array}$ & $\begin{array}{c}C_{1000}^{t o t} \\
{[€ / \mathrm{km}]}\end{array}$ & $\begin{array}{c}C_{1500}^{t o t} \\
{[€ / \mathrm{km}]}\end{array}$ & $\begin{array}{c}C_{2000}^{t o t} \\
{[€ / \mathrm{km}]}\end{array}$ \\
\hline Technology 1 & Area 1 & 1 & 2.67 & 3 & 3.33 \\
Technology 1 & Area 2 & 2 & 3.67 & 4 & 4.33 \\
Technology 1 & Area 3 & 3 & 4.67 & 5 & 5.33 \\
Technology 1 & Area 4 & 4 & 5.67 & 6 & 6.33 \\
\hline Technology 2 & Area 1 & 1 & 2.67 & 3 & 3.33 \\
Technology 2 & Area 2 & 2 & 3.67 & 4 & 4.33 \\
Technology 2 & Area 3 & 1 & 2.67 & 3 & 3.33 \\
Technology 2 & Area 4 & 2 & 3.67 & 4 & 4.33 \\
\hline
\end{tabular}

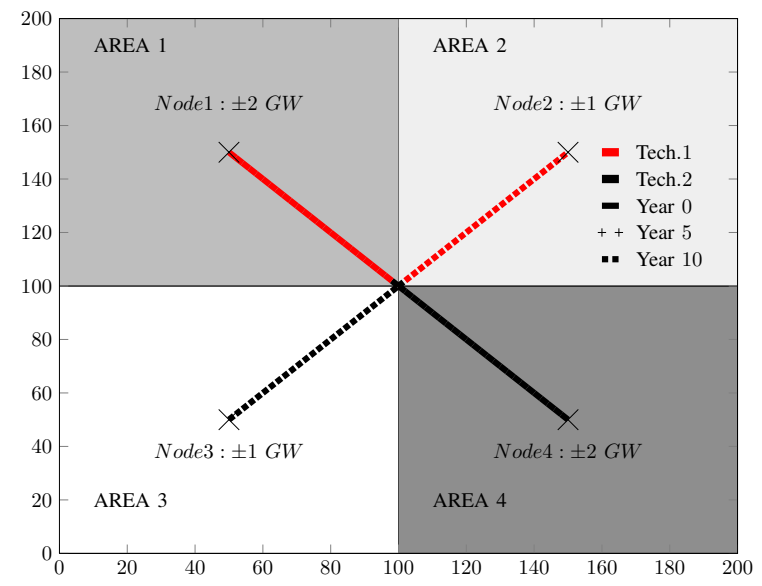

Fig. 7: Stepwise investment plan for case 1: base case

The optimization is performed for three time steps in years 0,5 and 10 . In year 0 , at least $1 \mathrm{GW}$ needs to be accomplished between both transmission zones. In years 5 and 10, the total capacity should be at least $2 \mathrm{GW}$ and $3 \mathrm{GW}$ respectively. A discount rate of $5 \%$ is used to account for depreciation.

Four different cases are analysed to show the working principle of the methodology. In case 1, which is the base case, only constraints regarding maximum power injection capabilities and maximum power per path are considered. The stepwise investment plan for case 1 is shown in Fig. 7. In year 0 of the investment time horizon, a $2 \mathrm{GW}$ link between nodes 1 and 4 is established. Technology 1 is used in Area 1, where it is cheapest. In Area 4, technology 2 is preferred. At year 5 of the investment time horizon, no new connection is built. In this case it is cheaper to oversize the connection between nodes 1 and 4 in year 0 . In the last step of the planning horizon, a $1 \mathrm{GW}$ link is established between nodes 2 and 4 . In Area 2 , technology 1 is used, whereas technology 2 is preferred for Area 3. The total costs are given in Tab. II. Further, the costs per time step (non-discounted and discounted costs), the calculation time and number of iterations of the developed methodology, the calculation time using brute force approach to determine global optimum and the net present value (NPV) of the global optimum are provided in Tab. II. Appendix C provides extra information about convergence behaviour between the MILP routine and the optimal routing calculation. The base case which only considers maximum power injection capabilities is obviously the cheapest possible option. All other cases put additional constraints on the optimization problem such that the obtained costs can only be equal to or higher than the costs of the base case. In the base case, $267.3 €$ of net present value were invested in year 10 .

In case 2, the available capital of year 10 is limited to $200 €$. Fig. 8 shows the optimal investment plan in this case. In year 0 , a $1 \mathrm{GW}$ connection is established between nodes 1 and 3 . In year 5 , a $2 \mathrm{GW}$ connection is built between nodes 2 and 4. As the power injection capability of node 2 is only $1 \mathrm{GW}$, another transmission link of $1 \mathrm{GW}$ between nodes 1 and 2 is needed. Tab. II shows that the total NPV of case 2 is 38.2 $€(4.73 \%)$ higher than in the base case.

In case 3 , the maximum amount of investments of year 0 
TABLE II

TOTAL COSTS AND CALCULATION TIMES FOR THE DEVELOPED METHODOLOGY AND GLOBAL OPTIMUM

\begin{tabular}{lccccccc} 
Case & Year & $\begin{array}{c}\text { Costs } \\
{[€]}\end{array}$ & $\begin{array}{c}\text { Discounted costs } \\
{[€]}\end{array}$ & $\begin{array}{c}t_{\text {calc }} \\
{[\mathrm{s}]}\end{array}$ & $\begin{array}{c}N_{\text {iter }} \\
{\left[\begin{array}{c}t_{\text {calc }}^{\text {global }} \\
{[\mathrm{s}]}\end{array}\right.}\end{array}$ & $\begin{array}{c}\text { Global optimum } \\
{[€]}\end{array}$ \\
\hline Case 1 & 0 & 540,7 & 540.7 & - & - & - & - \\
Case 1 & 5 & 0 & 0 & - & - & - & - \\
Case 1 & Year 10 & 446.3 & 267.3 & - & - & - & - \\
Case 1 & Total NPV & - & $\mathbf{8 0 8}$ & 399.7 & 10 & 1924.2 & $\mathbf{8 0 8}$ \\
\hline Case 2 & Year 0 & 266.7 & 266.7 & - & - & - & - \\
Case 2 & Year 5 & 749 & 579.5 & - & - & - & - \\
Case 2 & Year 10 & 0 & 0 & - & - & - & - \\
Case 2 & Total NPV & - & $\mathbf{8 4 6 . 2}$ & 443.6 & 10 & 1932.2 & $\mathbf{8 4 6 . 2}$ \\
\hline Case 3 & Year 0 & 266.7 & 266.7 & - & - & - & - \\
Case 3 & Year 5 & 366.7 & 283.7 & - & - & - & - \\
Case 3 & Year 10 & 446.3 & 267.3 & - & - & - & - \\
Case 3 & Total NPV & - & $\mathbf{8 1 7 . 7}$ & 438.8 & 10 & 1894 & $\mathbf{8 1 7 . 7}$ \\
\hline Case 4 & Year 0 & 366.7 & 366.7 & - & - & - & - \\
Case 4 & Year 5 & 266.7 & 206.3 & - & - & - & - \\
Case 4 & Year 10 & 446.3 & 267.3 & - & - & - & - \\
Case 4 & Total NPV & - & $\mathbf{8 4 0 . 3}$ & 392 & 10 & 1866 & $\mathbf{8 4 0 . 3}$ \\
\hline
\end{tabular}

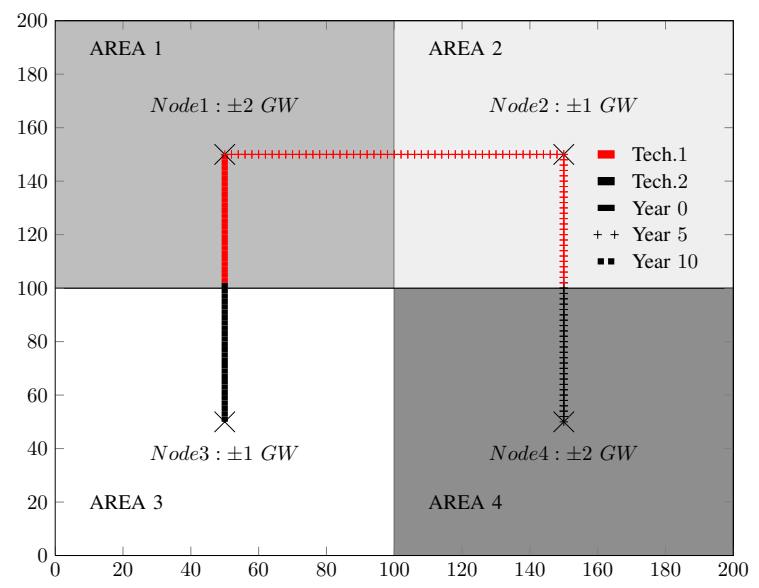

Fig. 8: Stepwise investment plan for case 2: capital restriction

are limited to $400 €$. The maximum investment constraint of year 10 is relaxed again. In this case, we can see that in all investment time steps connections of $1 \mathrm{GW}$ are established (Fig. 9). In year 0 , node 1 is connected with node 3 . In year 5 , nodes 2 and 4 are connected. In year 10, node 1 is connected with node 4 . In this case, the most expensive connection is established at the latest stage of the planning horizon as the depreciation is the highest. Still, in case 3, the NPV of the total investments are $9.7 €$ higher than in the base case (Tab. II).

In case 4 , the connections between nodes 1 and 3 as well as between nodes 1 and 4 are not allowed before year 5 . In this case, we can see that in year 0 , nodes 2 and 4 are connected with a $1 \mathrm{GW}$ link which is the cheapest option for this case. In year 5 , the connection of nodes 1 and 3 is established and in year 10, nodes 1 and 4 are connected (Fig.10). In fact, the same grid topology is obtained as in case 3, except that the order of investments in year 0 and 5 are switched. In this case, the costs are $22.6 €$ higher than in case 3 , as the the more expensive link between nodes 2 and 4 has to be built in year 0 and as such cannot be depreciated.

Tab. II shows that the global optimum is reached in all investigated cases with a significantly shorter computation time. Tab. II also shows that the calculation time depends mainly on the optimal routing algorithm, rather than the MILP part of the process. In the brute force case, the minimal costs of each possible path and power rating are calculated resulting

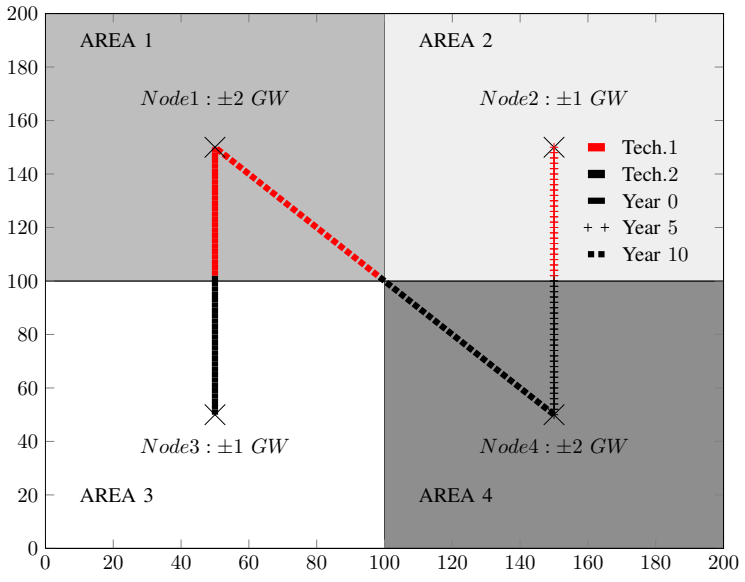

Fig. 9: Stepwise investment plan for case 3: limitation on maximum investment by owner

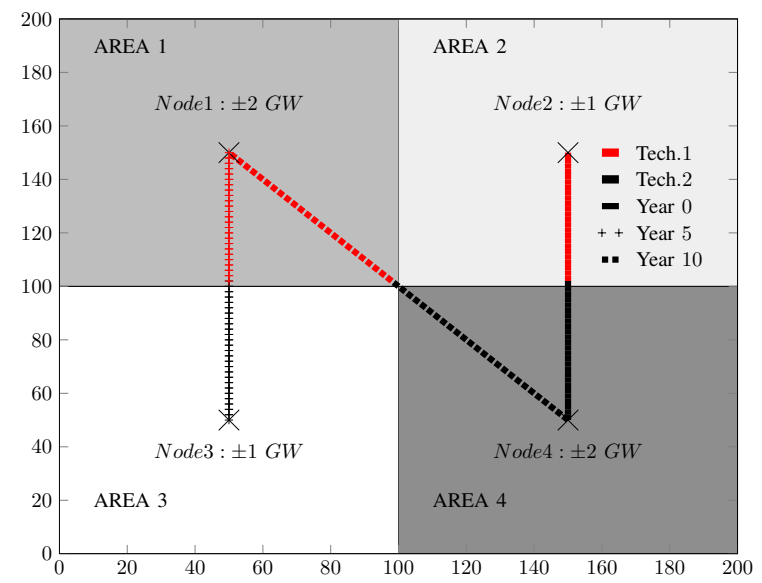

Fig. 10: Stepwise investment plan for case 4: delay of corridor availability

in 180 optimal routing calculations (6 possible paths and 30 possible ratings). In that case, the MILP problem is solved only once. In the iterative approach, the optimal routing algorithm is only used links and power ratings selected by the MILP optimization of the first iteration. The costs of chosen paths and ratings are updated for the next iteration.

In the following section, the developed methodology is applied to a larger case study and the effect of different constraints on the obtained results is discussed qualitatively.

\section{APPLiCATION to A LARGER SYSTEM}

In this section, the developed methodology is applied to a case study to show possibilities of application and the capabilities of the methodology. The selected case study aims to analyse different scenarios for long term and large scale transmission system investments. The starting point of the case study is the roadmap for a low-carbon economy in Europe for 2050 established by the European Climate Foundation [61], [62]. The roadmap states that by 2050 additional $33 \mathrm{GW}$ of transmission capacity between the Iberian peninsula and France would enable economically a more efficient use of for power generation, especially solar and wind resources [61].

In order to determine area dependent installation costs, geographic information about the Iberian peninsula and France has 


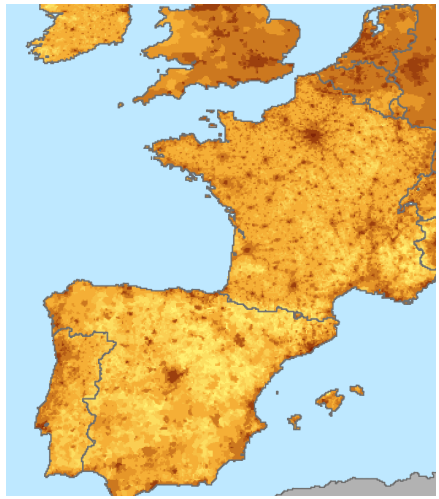

(a) Used population density map [63]

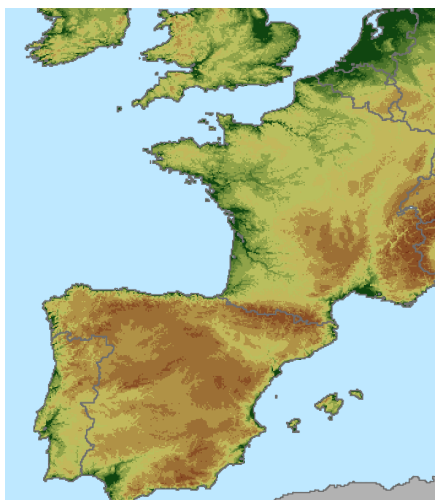

(b) Used elevation map [64]

Fig. 11: Population density and elevation map of the Iberian peninsula and France

been derived from the Socio-economic Data and Applications Centre. In order to weight social impact, a population density map is used whereas an elevation map is used to reflect the topographic properties of the area of focus (Fig. 11). The total spatial weight $\mathcal{W}_{\text {tot }}$ is determined by combining the information of both maps

$$
\mathcal{W}_{\text {tot }}=\frac{a_{e} \cdot \mathcal{W}_{\text {elevation }}+a_{p} \cdot \mathcal{W}_{\text {population }}}{a_{e}+a_{p}}
$$

where, $a_{e}$ and $a_{p}$ indicate how much the elevation and population density contribute to the total spatial weight $\mathcal{W}_{\text {tot }}$. Tab. III shows optimal solutions for different combinations of $a_{e}$ and $a_{p}$.

The European transmission grid model according [65] provides a sufficiently accurate representation of the European high voltage transmission grid. To limit calculation time, only the Spanish and French transmission grids are used in the calculation of maximum power injection capabilities (Fig. 12). The neighbouring transmission grids of Portugal, Italy, Germany, Switzerland, Belgium and UK have been represented using equivalent injections on border nodes. A total of 34 candidate nodes have been selected depending on their injection capabilities and locations. As shown in Fig. 12, candidate nodes have been geographically spread in both countries. Out of the 34 candidate nodes, 18 are situated in France whereas 16 candidate nodes have been selected in Spain.

The probability distribution of loads is simulated by scaling hourly load values of the Spanish and French transmission grids available in [66], [67] to the transmission grid model used and using the demand values provided in [65] as the mean values of the probability distribution. The generation profile of wind farms is simulated using randomly generated and Weibull distributed wind speeds and applying them to a wind farm power output curve as given in [68] and using the provided generation values of [65] as maximum generation values.

Fig. 13 shows the obtained power injection and absorption capabilities after application of the calculation methodology provided in [56], [57]. The figure shows that in nodes 26, 28 and 31 the most amount of power can be imported whereas

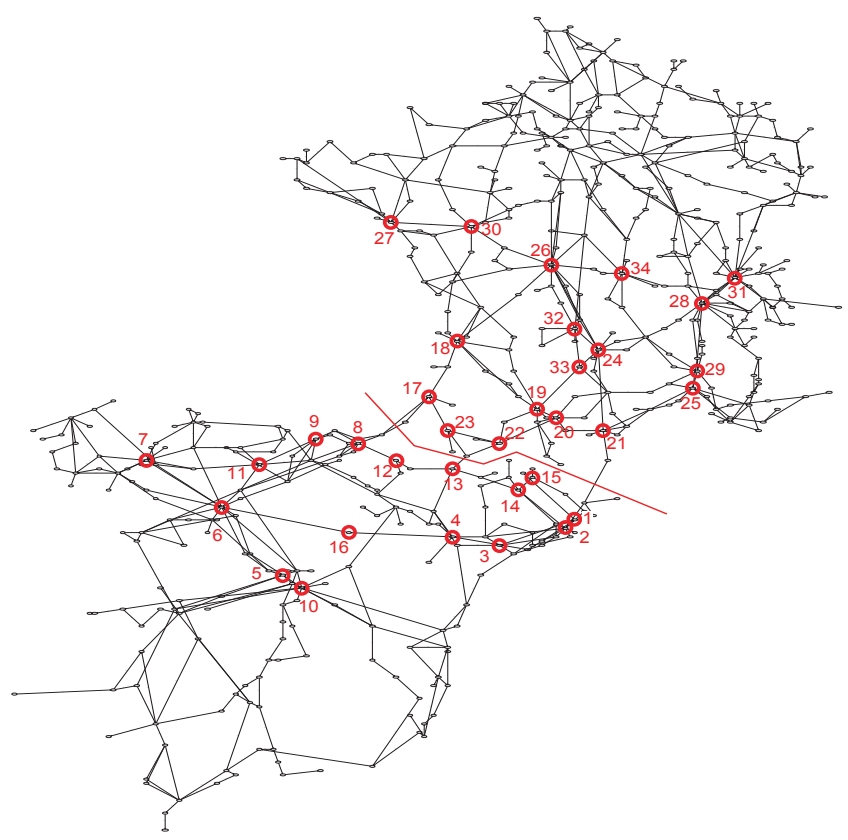

Fig. 12: Structure of the used network. Circles indicate chosen candidate nodes [65]

nodes $6,10,26,28$ have the highest power export capacity.

The following paragraphs show possible future grid expansion options to establish $30 \mathrm{GW}$ of additional interconnection capacity between Spain and France. Following optimization parameters remain unchanged in all scenarios:

- The total interconnection power is established in four investment time steps, $\mathbf{t}=\left[\begin{array}{llll}0 & 10 & 20 & 30\end{array}\right]$ years

- The interconnection power per time step is $P_{t}^{\text {inter }}=$ [8 $\left.\begin{array}{llll}8 & 16 & 23 & 30\end{array}\right] \mathrm{GW}$

- The new connections can be built in multiples of $500 \mathrm{MW}$

- The used discount rate is $3 \%$ per year, without accounting for inflation and price increase of equipment

Fig. 14 shows the optimal investment plan if the maximum power rating per path $\overline{\mathbf{K}}$ is restricted to $3 \mathrm{GW}$. In the first investment time step, most investments are established using HVAC technology (Fig. 14). Candidate nodes in both zones which are closest to each other are connected first. With advancing time, the total length of new built connections increase. In this scenario, $1301 \mathrm{~km}, 1530 \mathrm{~km}, 1658 \mathrm{~km}$ and $2835 \mathrm{~km}$ of new lines are built at each time step. Under the taken assumptions, most interconnections are established using overhead line technology. During the entire planning horizon, only one underground cable connection appears using HVDC technology (Fig. 14b). HVAC technology is preferred to connect nodes close to each other, whereas for longer distances HVDC technology is used. As HVDC overhead lines require less conductors, the cost per km of HVDC overhead line is less than HVAC overhead lines. With increasing transmission distance, cost savings of using HVDC overhead lines overweights costs of HVDC converters making HVDC economically more feasible.

The total net present value of the grid expansion equals $8224 \mathrm{M} €$. If the costs are broken down by time step, we can 


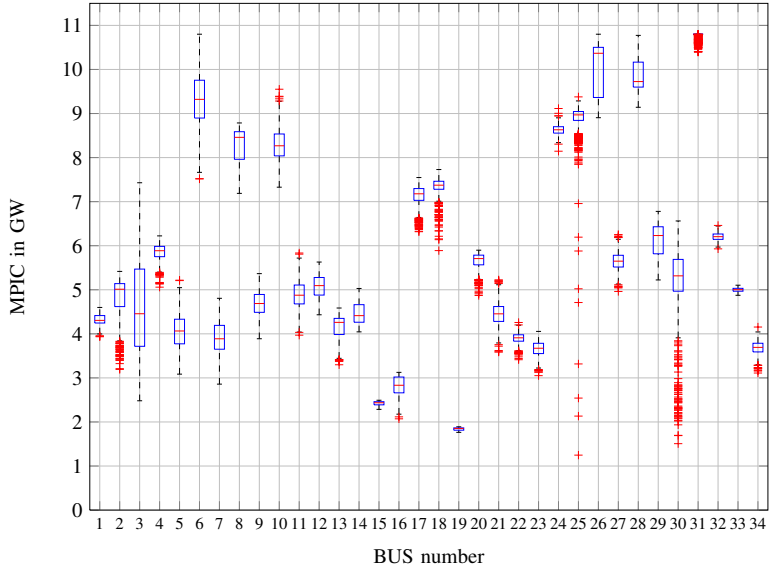

(a) Maximum power import capabilities of candidate nodes

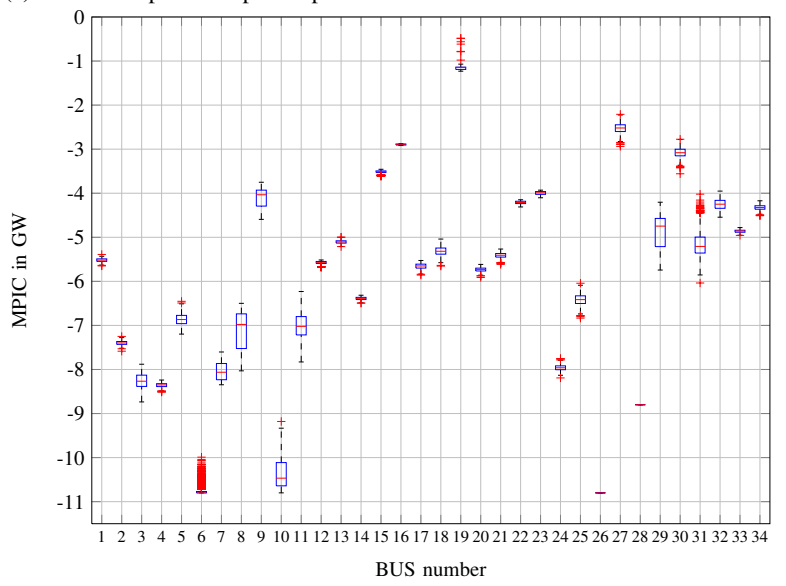

(b) Maximum power export capabilities of candidate nodes

Fig. 13: Maximum power import and export capabilities of candidate nodes

see that discounted investments worth $1895 \mathrm{M} €, 2138 \mathrm{M} €$, $1864 \mathrm{M} €, 2327 \mathrm{M} €$ are required in the four time steps. The net present value is spread approximately equally over the planning horizon.

Fig. 15 shows the optimal investment plan if links with a maximum rating of $5 \mathrm{GW}$ can be established. In this case, the number of necessary links is reduced to 15 instead of 17 . Tab. III shows that the total investment cost is decreased if transmission links with $5 \mathrm{GW}$ are used. The total investments decrease to $7408 \mathrm{G} €$ equal to a cost saving of $9.91 \%$. Tab. III further shows that the required length of new lines is approximately $2000 \mathrm{~km}$ less if links with a capacity of $5 \mathrm{GW}$ are used. This has a positive impact on maintenance decreasing operational costs. Under the assumptions, only overhead lines are used in this configuration.

Although any link can be built with a rating of $5 \mathrm{GW}$, only one transmission link is actually built with a capacity of $5 \mathrm{GW}$ connecting nodes 4 and 33 via an HVDC overhead line. The reason is that the use of larger links is limited by the maximum power injection capability of candidate nodes. In case larger links would be connected, overload situations in the underlying network may occur. Tab. III shows that a further increase in maximum link capacities does not improve the optimal solution. Even if links with a capacity of $8 \mathrm{GW}$ are allowed, the economically best solution is still achieved
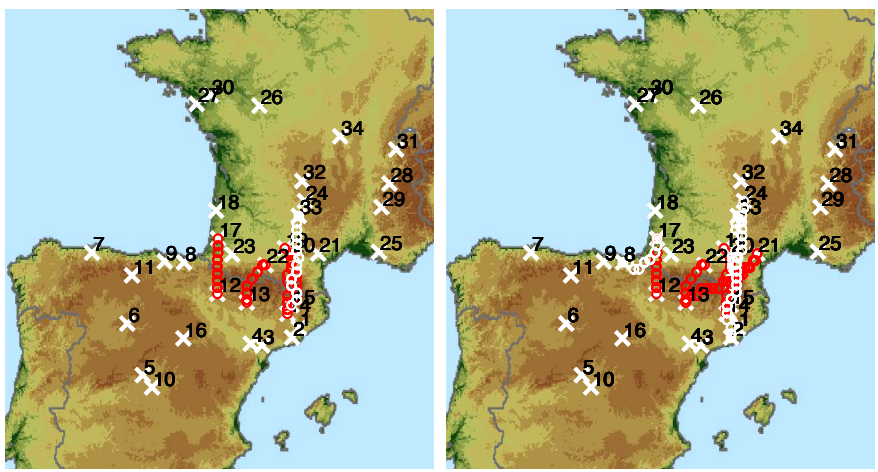

(a) Optimal grid expansion $t=0$ (b) Optimal grid expansion $t=10$ years

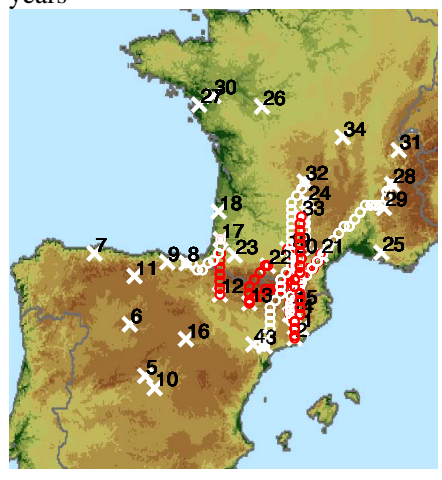
years

(c) Optimal grid expansion $t=20$ (d) Optimal grid expansion $t=30$ years years

Fig. 14: Optimal investment plan for $\overline{\mathbf{K}}=3 G W$. Red colour indicates HVAC, white colour indicates HVDC. Solid lines indicate underground cables, circles indicate overhead lines

using $5 \mathrm{GW}$.

Tab. III shows the optimal solutions in case the multiterminal HVDC constraint and spatial weights are varied. Significant cost savings can be achieved if multi-terminal operation of HVDC links becomes possible in early stages of the planning process. The table shows that similar solutions are achieved when spatial weights are varied.

\section{TABLE III}

COMPARISON OF DIFFERENT MAXIMUM POWER RATINGS PER PATH

\begin{tabular}{|c|c|c|c|c|c|c|}
\hline$\overline{\mathbf{K}}$ & \multicolumn{3}{|c|}{$C_{t o t}[M €]$} & \multicolumn{3}{|c|}{$l_{\text {tot }}[\mathrm{km}]$} \\
\hline $3000 \mathrm{GW}$ & & & & & & \\
\hline$a_{e}=2, a_{p}=1$ & 8224 & 10920 & 11387 & 7054 & 9517 & 8939 \\
\hline$a_{e}=1, a_{p}=1$ & 7898 & 8010 & 8179 & 6789 & 6846 & 7334 \\
\hline$a_{e}=1, a_{p}=2$ & 8220 & 8220 & 8536 & 7376 & 7376 & 7034 \\
\hline $5000 \mathrm{GW}$ & & & & & & \\
\hline$a_{e}=2, a_{p}=1$ & 7408 & 7629 & 8446 & 5098 & 4840 & 5846 \\
\hline$a_{e}=1, a_{p}=1$ & 7469 & 7570 & 7756 & 5332 & 4688 & 5209 \\
\hline$a_{e}=1, a_{p}=2$ & 7548 & 7548 & 7755 & 4913 & 4913 & 5291 \\
\hline $8000 \mathrm{GW}$ & & & & & & \\
\hline$a_{e}=2, a_{p}=1$ & 7408 & 7629 & 8061 & 5098 & 4840 & 4847 \\
\hline$a_{e}=1, a_{p}=1$ & 7469 & 7570 & 7756 & 5332 & 4688 & 5209 \\
\hline$a_{e}=1, a_{p}=2$ & 7548 & 7548 & 7755 & 4913 & 4913 & 5291 \\
\hline
\end{tabular}

The optimal solution obtained with transmission paths of $5 \mathrm{GW}$ contains 4 HVAC connections established in the first time step of the planning horizon between nodes 12 and 17, 12 and 23, 14 and 20,13 and 22 (Fig. 15a). In case these connections cannot be built in the first 10 years, the investment plan shown in Fig. 16 becomes economically more feasible. In this option, the connections between nodes 12 and 17 , 14 and 20,13 and 22 are established in the second step of 

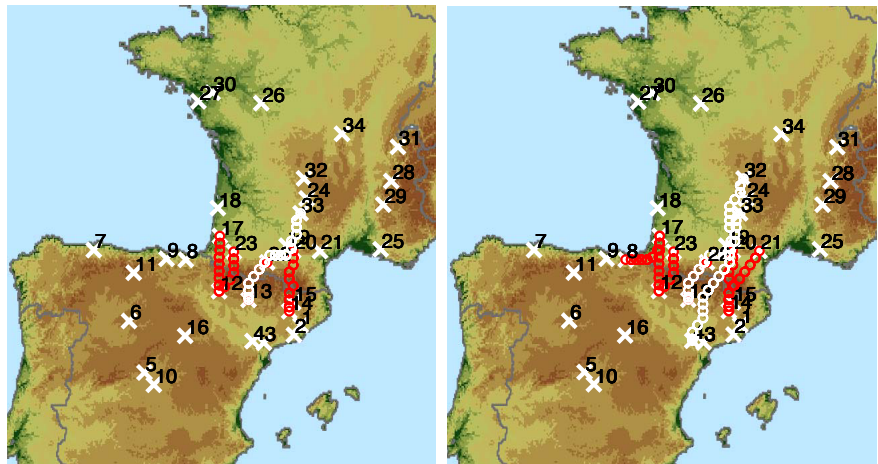

(a) Optimal grid expansion $t=0$ (b) Optimal grid expansion $t=10$ years

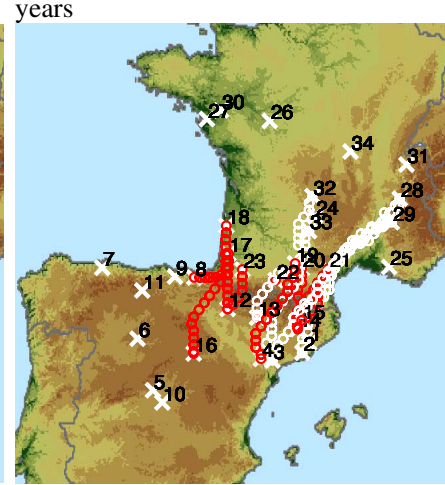

(c) Optimal grid expansion $t=20$ (d) Optimal grid expansion $t=30$ years years

Fig. 15: Optimal investment plan for $\overline{\mathbf{K}}=5 G W$. Red colour indicates HVAC, white colour indicates HVDC. Solid lines indicate underground cables, circles indicate overhead lines

the planning horizon. The connection between nodes 12 and 23 is not established at all. The total net present value of investments considering line delays equals $7515 \mathrm{M} €$, which is $1.01 \%$ higher than the solution without delay. In the optimal investment plan including line delays, 13 transmission paths are established instead of 15 . The total length of new built lines is approximately $300 \mathrm{~km}$ less. In this case, a detailed analysis of expected transmission losses and maintenance costs should be conducted in order to assess potential cost savings due to shorter lines versus the $1.01 \%$ investment cost increase.

\section{CONCLUSION}

This paper proposes a transmission system expansion optimization methodology considering temporal and spatial aspects. The methodology delivers the optimal time point, location, rating, technology and routing of new investments. This methodology can be implemented by transmission system operators to determine optimal grid expansion plans for different future scenario's. The different building blocks of the methodology can be included in existing transmission planning tools or used as a standalone tool as demonstrated in this paper.

The calculation results confirm that in the early time steps links with shorter distances and lower power ratings are preferred to realise a certain interconnection capacity. The more expensive long distance and high power links are postponed as long as possible. For the long distance and high power transmission links, mostly HVDC technology is preferred as
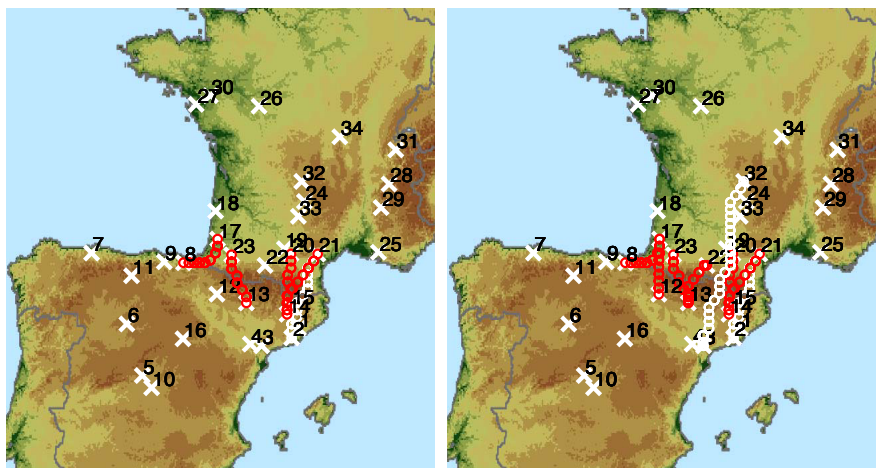

(a) Optimal grid expansion $t=0$ (b) Optimal grid expansion $t=10$ years

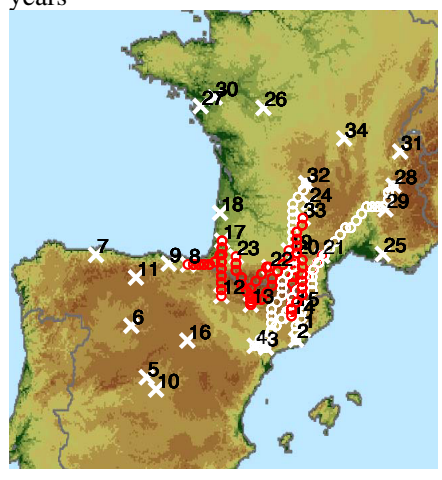
years

(c) Optimal grid expansion $t=20$ (d) Optimal grid expansion $t=30$ years years

Fig. 16: Optimal investment plan for $\overline{\mathbf{K}}=5 G W$ in case of line delays. Red colour indicates HVAC, white colour indicates HVDC. Solid lines indicate underground cables, circles indicate overhead lines

the cost savings of cheaper transmission lines overweight the additional HVDC converter costs.

Cost savings can be achieved using transmission links with higher capacities. Nevertheless, the underlying transmission grid sets limits to the maximum capacity of such links. Therefore, it is important to consider several scenarios regarding link capacity in order to determine the economically most beneficial option. Significant investment cost savings can be achieved if multi-terminal HVDC operation is technically feasible.

It should be noted that obtained results strongly depend on used data. It is important to perform sensitivity analysis on the used data and assumptions. Costs of different investment plans may be close to each other although the obtained grid topologies are very different. In such cases, the operational costs such as transmission system losses and maintenance costs can be decisive.

\section{APPENDIX A \\ Calculation of $P_{M P I C}$ and $\Delta_{M P I C}$}

This section is based on [57] and briefly explains the calculation of the $P_{M P I C}$ vector and the $\Delta_{M P I C}^{t o t}$ matrix. $P_{M P I C}$ illustrates the maximum power injection capabilities whereas $\Delta_{M P I C}^{t o t}$ expresses how the maximum power injection capability of certain nodes in the power system change due to injections in other nodes. $P_{M P I C}$ and $\Delta_{M P I C}^{t o t}$ are calculated using a DC optimal power flow approach taking the probabilis- 
tic nature of renewable generation and the behivor of loads into account.

The maximum power injection capability (MPIC) is calculated using the objective function in (18), $n_{g}$ being the number of generators, $f_{p}^{i}\left(p_{g}^{i}\right)$ the generation costs of generator $i$ as a function of its power injection, $p_{g}^{x}$ the power injection of the investigated node $x$ and $K$ a scaling factor to combine the two objectives of the optimization, namely the minimization of total generation costs and maximization of the injection in the investigated node $x$. The optimization constraints are the flow limits of transmission lines, voltage magnitudes, voltage angle limits, active and reactive power limits of generators, the Kirchhoff equations and the system load-generation balance. In case the absorption capability is calculated, $p_{g}^{x}$ can only be negative, implying that a negative value of $K$ needs to be used.

$$
\min \sum_{i=1}^{n_{g}} f_{p}^{i}\left(p_{g}^{i}\right)-K p_{g}^{x}
$$

Using probabilistic load and generation profiles and varying node $x$ between all nodes in the system, a range of possible maximum power injections can be obtained for each node. Fig. 13 shows such an example .

Let us consider a node $x^{1}$. To see the change in the MPIC of node $x^{1}$ depending on the injection in another node $x^{2}$, a generator is added in node $x^{2}$. The generator has negative production costs so it will be selected by the OPF algorithm described above. The power rating of the generator is varied among all possible power ratings used in the MILP optimization. This way, the change in the MPIC of node $x^{1}$ depending on the injection in node $x^{2}$ is calculated $\left(\Delta_{1,2,1}\right)$. In case of absorptions, the additional generators are replaced by loads. (19) shows the structure of $\Delta_{M P I C}^{t o t}$. The first index indicates the affected node, the second index indicates the effecting nodes and the third index indicates the power rating.

$$
\Delta_{M P I C}^{t o t}=\left[\begin{array}{ccccccc}
\Delta_{1,1,1} & \Delta_{1,2,1} & \ldots & \Delta_{1, N, 1} & \ldots & \Delta_{1, N, N} & \\
\Delta_{2,1,1} & \Delta_{2,2,1} & \ldots & \Delta_{2, N, 1} & \ldots & \Delta_{2, N, N} & \\
\vdots & \vdots & \vdots & \vdots & \vdots & \vdots & \vdots \\
\Delta_{N, 1,1} & \Delta_{N, 2,1} & \ldots & \Delta_{N, N, 1} & \ldots & \Delta_{N, N, N} &
\end{array}\right]
$$

\section{APPENDIX B}

\section{A* SHORTEST PATH ALGORITHM}

The heuristic $A^{*}$ shortest path algorithm determines the shortest path between a starting point $X$ and an end point $Y$ by estimating the distance between a set A of neighbouring candidate nodes and the end point [60]. As a greedy algorithm that selects the first best answer in each step, $A^{*}$ can be faster than the Dijkstra approach.

Starting from $X$, the initial set $A$ of nodes are those directly connected to $X$. Next, the algorithm determines a distance estimate:

$$
h(X, Y)=d(X, A)+h(A, Y)
$$

$d(X, A)$ is the known distance between node $X$ and the nodes in set $A . h(A, Y)$ is an estimate of the distance between the nodes in set $A$ and the end point $Y$.
From the nodes in set $A$, the one with the lowest distance estimate $h(X, Y)$ is chosen as the next node $X_{n e x t}$ in the path. The actual distances of all other nodes in set $A$ to the starting point are saved in set $B$. These distances are the minima. In the next iteration, $X_{\text {next }}$ is defined as the new starting point and set $A$ becomes the set of nodes directly connected to $X_{n e x t}$. The estimates are recalculated for the nodes in set $A$. As some nodes of set $A$ can be part of set $B$, also the new estimates for these nodes are calculated using the distances in the separate set.

As long $h(A, Y)$ underestimates the distance between the actual node in set $A$ and the end node (e.g. by using the Euclidean distance), the algorithm converges to the shortest possible path. The $\mathrm{A}^{*}$ algorithm performs faster if the weights of the graph edges have a large difference as the estimate $h(A, Y)$ has similar values for all nodes in set $A$ if the Euclidean distance is used. The algorithm also performs faster if $h(A, Y)$ overestimates the distance between node $\mathrm{A}$ and the end node Y. In this case there is no guarantee of finding the shortest path.

\section{APPENDIX C}

\section{CONVERGENCE BEHAVIOUR}

Tab. IV shows the chosen power ratings per iteration for case 1 of the concept case shown in Section V. It is shown that the optimal solution is first found in iteration 2. As the convergence criterion is not met (Fig. 18) the penalty functions as given in (1) are applied and the next iteration is performed. Thus, the algorithm continues searching and finds several other solutions until iteration 10 where the difference of costs used for the MILP optimization and obtained form the optimal routing algorithm are the same. At iteration 10, the same topology as in iteration 2 is obtained. For branches and power ratings which have been selected by the MILP routine in previous iterations, no penalty functions are applied. Hence, if a topology is found twice, the cost difference between the MILP optimization and the optimal routing routine are the same, leading to convergence and stopping the calculation.

\section{TABLE IV}

\section{CHOSEN POWER RATINGS IN EACH ITERATION FOR CASE 1}

\begin{tabular}{c|cccccccccc} 
Branch & Iteration & & & & & & & & & \\
& 1 & 2 & 3 & 4 & 5 & 6 & 7 & 8 & 9 & 10 \\
\hline $1-2$ & 0 & 0 & 900 & 0 & 0 & 1000 & 100 & 1100 & 0 & 0 \\
$1-3$ & 1000 & 0 & 1100 & 100 & 1200 & 1000 & 1000 & 1000 & 2000 & 0 \\
$1-4$ & 1000 & 2000 & 0 & 1900 & 800 & 0 & 900 & 100 & 0 & 2000 \\
$2-3$ & 0 & 1000 & 0 & 900 & 200 & 0 & 0 & 0 & 1000 & 1000 \\
$2-4$ & 1000 & 0 & 1900 & 100 & 1200 & 2000 & 1100 & 2100 & 0 & 0 \\
$3-4$ & 0 & 0 & 100 & 0 & 0 & 0 & 0 & 0 & 2000 & 0 \\
\hline
\end{tabular}

Fig. 17 shows the evolution of the average costs used in the MILP routine per iteration for case 1 of the concept case. For the sake of simplicity only the costs of branch 1-3 are shown which is one of the selected branches in the optimal solution. The figure shows that in early iterations the costs per MW behave linearly over the range of possible power ratings. As soon as a small power rating is chosen by the MILP algorithm (100 MW in iteration 4), the non-linearity of the cost function due to the fixed part of the cost component becomes visible. The costs calculated by the optimal routing algorithm are approximately 5 times higher than the initially 
assumed costs for that particular branch and power rating. The figure also shows that costs determined by the optimal routing algorithm for a specific power rating are not subject to the cost update with penalty functions, as they are the theoretically minimum cost for that particular branch and power rating. This effect can be seen 1t $1000 \mathrm{MW}$. In iteration 1 the branch is selected with a $1000 \mathrm{MW}$ rating (lower cross at $1000 \mathrm{MW}$ ). After calculating the costs with the optima routing algorithm higher costs are obtained for this power rating (higher cross at $1000 \mathrm{MW})$. From iteration 2 onwards all cost functions go through this point as no penalty functions are applied to that point.

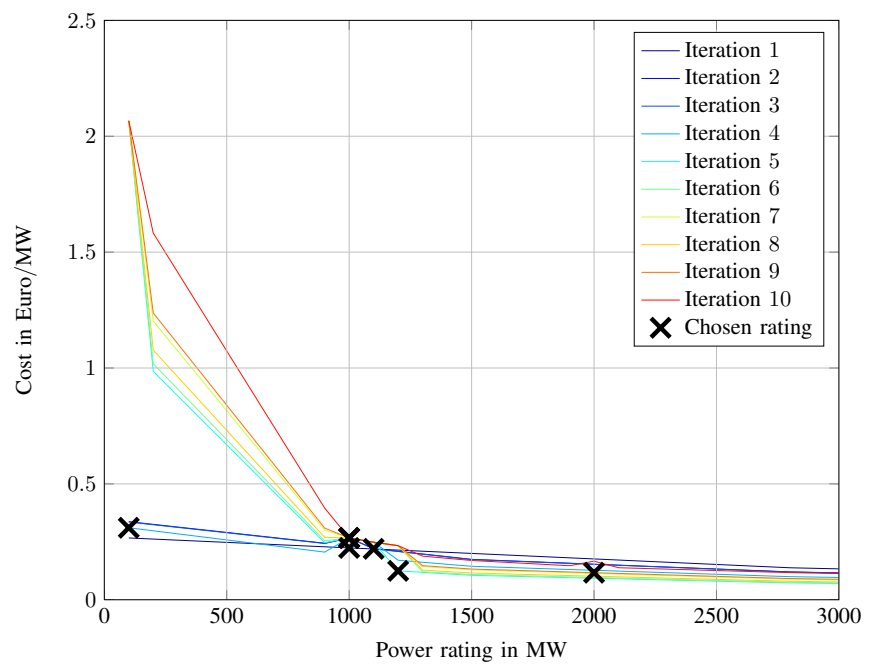

Fig. 17: Evolution of the costs for branch 1-3 over the iterations for case 1 of concept case

Fig.18 shows the relative difference between the objective function value obtained by the MILP algorithm and the costs calculated with the optimal routing algorithm for case 1 of the concept case. The figure shows that the total costs are underestimated by approximately $18 \%$ in iteration 2 where the optimal solution is found for the first time. As there is a cost difference between the both solutions, the algorithm applies the aforementioned penalty functions and carries on with the search. We can see that after iteration 8 there is a steady decrease in the relative cost difference and eventually eventually leading to convergence and finding the optimal solution in iteration 10 .

\section{REFERENCES}

[1] Eurelectric Facts Database. Maximum Net Generation Capacity by Primary Energy. http://www.eurelectric.org/factsfolders/factsfolder.asp? FolderID $=5245$.

[2] European Wind Energy Association. (2015, February) Wind in Power - 2014 European Statistics. http://www.ewea.org/fileadmin/files/library/ publications/statistics/EWEA-Annual-Statistics-2014.pdf.

[3] Entso-e. Ten Year Network Development Plan 2012 https://www.entsoe.eu/fileadmin/user_upload/_library/SDC/TYNDP/ 2012/TYNDP_2012_report.pdf.

[4] G. Latorre, R. Cruz, J. Areiza, and A. Villegas, "Classification of Publications and Models on Transmission Expansion Planning," Power Systems, IEEE Transactions on, vol. 18, no. 2, pp. 938 - 946, may 2003

[5] C. Lee, S. Ng, J. Zhong, and F. Wu, "Transmission expansion planning from past to future," in Power Systems Conference and Exposition, 2006. PSCE '06. 2006 IEEE PES, Oct 2006, pp. 257-265.

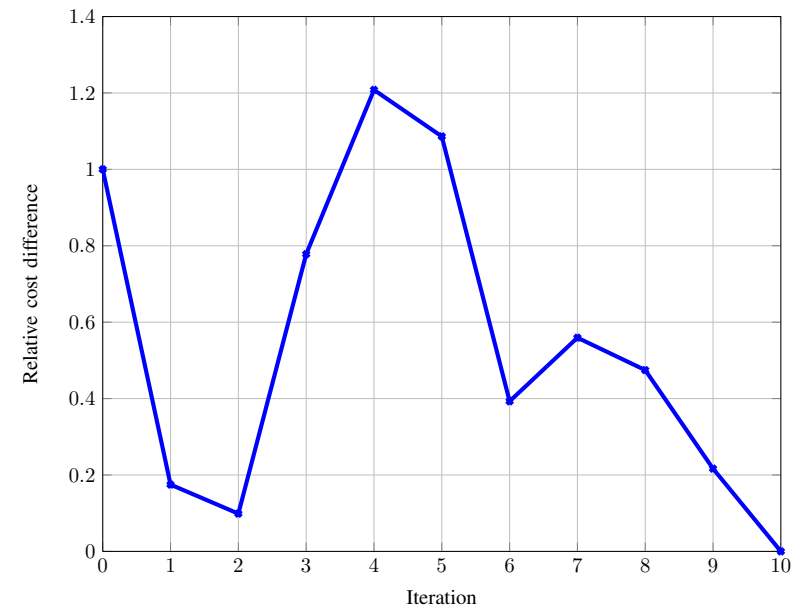

Fig. 18: Evolution of the relative cost difference used in MILP and obtained by optimal routing algorithm for the concept case

[6] L. Garver, "Transmission Network Estimation Using Linear Programming," Power Apparatus and Systems, IEEE Transactions on, vol. PAS89, no. 7, pp. $1688-1697$, sept. 1970.

[7] V. Levi and M. Calovic, "A new decomposition based method for optimal expansion planning of large transmission networks," Power Systems, IEEE Transactions on, vol. 6, no. 3, pp. 937 -943, aug 1991.

[8] R. Chanda and P. Bhattacharjee, "Application of computer software in transmission expansion planning using variable load structure," Electric Power Systems Research, vol. 31, no. 1, pp. 13 - 20, 1994.

[9] H. Youssef and R. Hackam, "New transmission planning model," Power Systems, IEEE Transactions on, vol. 4, no. 1, pp. 9 -18, feb 1989.

[10] J. Choi, T. D. Mount, and R. J. Thomas, "Transmission expansion planning using contingency criteria," Power Systems, IEEE Transactions on, vol. 22, no. 4, pp. 2249-2261, 2007.

[11] S. de la Torre, A. Conejo, and J. Contreras, "Transmission expansion planning in electricity markets," Power Systems, IEEE Transactions on, vol. 23, no. 1, pp. 238-248, Feb 2008.

[12] L. Bahiense, G. Oliveira, M. Pereira, and S. Granville, "A mixed integer disjunctive model for transmission network expansion," Power Systems, IEEE Transactions on, vol. 16, no. 3, pp. 560 -565, aug 2001.

[13] S. Haffner, A. Monticelli, A. Garcia, J. Mantovani, and R. Romero, "Branch and bound algorithm for transmission system expansion planning using a transportation model," Generation, Transmission and Distribution, IEE Proceedings-, vol. 147, no. 3, pp. 149 -156, may 2000.

[14] A. Kazerooni and J. Mutale, "Transmission network planning under security and environmental constraints," Power Systems, IEEE Transactions on, vol. 25, no. 2, pp. 1169-1178, May 2010

[15] N. Gupta, R. Shekhar, and P. K. Kalra, "Congestion management based roulette wheel simulation for optimal capacity selection: Probabilistic transmission expansion planning," International Journal of Electrical Power \& Energy Systems, vol. 43, no. 1, pp. 1259 - 1266 , 2012. [Online]. Available: http://www.sciencedirect.com/science/article/ pii/S0142061512003080

[16] M. Rider, A. Garcia, and R. Romero, "Power system transmission network expansion planning using ac model," IET Generation, Transmission \& Distribution, vol. 1, no. 5, pp. 731-742, 2007.

[17] E. J. De Oliveira, I. C. d. Silva Jr, J. L. R. Pereira, and S. Carneiro Jr, "Transmission system expansion planning using a sigmoid function to handle integer investment variables," Power Systems, IEEE Transactions on, vol. 20, no. 3, pp. 1616-1621, 2005.

[18] A. Ekwue and B. Cory, "Transmission System Expansion Planning by Interactive Methods," Power Apparatus and Systems, IEEE Transactions on, vol. PAS-103, no. 7, pp. 1583 -1591, july 1984.

[19] A. Monticelli, A. Santos, M. Pereira, S. Cunha, B. Parker, and J. Praca, "Interactive Transmission Network Planning Using a Least-Effort Criterion," Power Apparatus and Systems, IEEE Transactions on, vol. PAS101 , no. 10 , pp. $3919-3925$, oct. 1982.

[20] I. d. J. Silva, M. Rider, R. Romero, A. Garcia, and C. Murari, "Transmission network expansion planning with security constraints," IEE Proceedings-Generation, Transmission and Distribution, vol. 152, no. 6 , pp. 828-836, 2005. 
[21] P. Maghouli, S. Hosseini, M. Buygi, and M. Shahidehpour, "A multiobjective framework for transmission expansion planning in deregulated environments," Power Systems, IEEE Transactions on, vol. 24, no. 2, pp. 1051-1061, May 2009.

[22] A. Dominguez, A. Escobar Z, and R. Gallego, "Transmission expansion planning considering conductor proposals with different wire size and technology," in Transmission Distribution Conference and Exposition Latin America (PES T D-LA), 2014 IEEE PES, Sept 2014, pp. 1-6.

[23] M. Rahmani, M. Rashidinejad, E. Carreno, and R. Romero, "Efficient method for $\{\mathrm{AC}\}$ transmission network expansion planning," Electric Power Systems Research, vol. 80, no. 9, pp. 1056 - 1064 2010. [Online]. Available: http://www.sciencedirect.com/science/article/ pii/S0378779610000398

[24] A. Escobar, R. A. Gallego, R. L. Romero, and S. A. de Oliveira, "Hybrid model analysis and constructive algorithms in the performance of a genetic algorithm for the planning of an electric power transmission system," Proc. 17th Int. Conf. CARS and FOF, 2001.

[25] H. Gil and E. da Silva, "A reliable approach for solving the transmission network expansion planning problem using genetic algorithms," Electric Power Systems Research, vol. 58, no. 1, pp. 45 - 51, 2001.

[26] R. Romero, R. Gallego, and A. Monticelli, "Transmission system expansion planning by simulated annealing," in Power Industry Computer Application Conference, 1995. Conference Proceedings., 1995 IEEE, may 1995 , pp. $278-283$.

[27] R. Gallego, A. Alves, A. Monticelli, and R. Romero, "Parallel simulated annealing applied to long term transmission network expansion planning," Power Systems, IEEE Transactions on, vol. 12, no. 1, pp. $181-188$, feb 1997.

[28] A. Braga and J. Saraiva, "A multiyear dynamic approach for transmission expansion planning and long-term marginal costs computation," Power Systems, IEEE Transactions on, vol. 20, no. 3, pp. 1631-1639, Aug 2005.

[29] R. Gallego, R. Romero, and A. Monticelli, "Tabu search algorithm for network synthesis," Power Systems, IEEE Transactions on, vol. 15, no. 2, pp. $490-495$, may 2000.

[30] E. Da Silva, J. Ortiz, G. De Oliveira, and S. Binato, "Transmission network expansion planning under a Tabu Search approach," Power Systems, IEEE Transactions on, vol. 16, no. 1, pp. 62 -68, feb 2001.

[31] M. AlRashidi and M. El-Hawary, "A survey of particle swarm optimization applications in electric power systems," Evolutionary Computation, IEEE Transactions on, vol. 13, no. 4, pp. 913-918, Aug 2009.

[32] Y. del Valle, G. Venayagamoorthy, S. Mohagheghi, J.-C. Hernandez, and R. Harley, "Particle swarm optimization: Basic concepts, variants and applications in power systems," Evolutionary Computation, IEEE Transactions on, vol. 12, no. 2, pp. 171-195, April 2008.

[33] Y. Dusonchet and A. El-Abiad, "Transmission Planning Using Discrete Dynamic Optimizing," Power Apparatus and Systems, IEEE Transactions on, vol. PAS-92, no. 4, pp. 1358 -1371, july 1973.

[34] J. Dodu and A. Merlin, "Dynamic Model for long-term Expansion Planning Studies of Power Transmission Systems: the Ortie Model,' International Journal of Electrical Power; Energy Systems, vol. 3, no. 1, pp. 2 - 16, 1981.

[35] J.-C. Kaltenbach, J. Peschon, and E. Gehrig, "A Mathematical Optimization Technique for the Expansion of Electric Power Transmission Systems," Power Apparatus and Systems, IEEE Transactions on, vol. PAS-89, no. 1, pp. $113-119$, jan. 1970.

[36] R.-C. Leou, "A multi-year transmission planning under a deregulated market," International Journal of Electrical Power \& Energy Systems, vol. 33, no. 3, pp. 708 - 714, 2011. [Online]. Available: http://www.sciencedirect.com/science/article/pii/S0142061511000147

[37] S. Kannan, S. Slochanal, and N. Padhy, "Application and comparison of metaheuristic techniques to generation expansion planning problem,' Power Systems, IEEE Transactions on, vol. 20, no. 1, pp. 466-475, Feb 2005.

[38] M. Sepasian, H. Seifi, A. Foroud, and A. Hatami, "A multiyear security constrained hybrid generation-transmission expansion planning algorithm including fuel supply costs," Power Systems, IEEE Transactions on, vol. 24, no. 3, pp. 1609-1618, Aug 2009.

[39] S. Sarhadi and T. Amraee, "Robust dynamic network expansion planning considering load uncertainty," International Journal of Electrical Power \& Energy Systems, vol. 71, no. 0, pp. 140 - 150, 2015. [Online]. Available: http://www.sciencedirect.com/science/article/ pii/S014206151500126X

[40] S. Binato and G. C. Oliveira, "A Heuristic Procedure to Cope with multiyear Transmission Expansion Planning," IEEE/KTH Stockholm Power Tech. Conf., Jun. 1995.
[41] S. Binato, G. de Oliveira, and J. de Araujo, "A greedy randomized adaptive search procedure for transmission expansion planning," Power Systems, IEEE Transactions on, vol. 16, no. 2, pp. 247 -253, may 2001.

[42] O. Azadegan, R. Hager, F. Leuthold, K. Reich, T. Schreier G. Layr, C. Todem, C. von Hirschhausen, O. Wadosch, and H. Weigt, "Price, Cost and Financial Aspects of Network Expansion in the European Context," RealiseGrid Project Report, Apr. 2010. [Online]. Available: http://realisegrid.rse-web.it/content/files/File/ Publications\%20and\%20results/Deliverable_REALISEGRID_3.2.2.pdf

[43] J. Contreras, "A cooperative game theory approach to transmission planning in power systems," Ph.D., Univ. California Berkeley, 1997.

[44] J. Contreras and F. Wu, "A kernel-oriented algorithm for transmission expansion planning," Power Systems, IEEE Transactions on, vol. 15, no. 4, pp. $1434-1440$, nov 2000.

[45] — "Coalition formation in transmission expansion planning," Power Systems, IEEE Transactions on, vol. 14, no. 3, pp. $1144-1152$, aug 1999.

[46] A. Orths and Z. Styczynski, "Game theoretical approach to power network planning," in Power Tech Proceedings, 2001 IEEE Porto, vol. 1, 2001, p. 6 pp. vol.1.

[47] W. Li and P. Choudhury, "Probabilistic transmission planning," Power and Energy Magazine, IEEE, vol. 5, no. 5, pp. 46-53, Sept 2007.

[48] P. Sanchez-Martin, A. Ramos, and J. Alonso, "Probabilistic midterm transmission planning in a liberalized market," Power Systems, IEEE Transactions on, vol. 20, no. 4, pp. 2135-2142, Nov 2005.

[49] H. Kakuta and H. Mori, "A multi-objective memetic algorithm for probabilistic transmission network expansion planning," in Systems Man and Cybernetics (SMC), 2010 IEEE International Conference on, Oct 2010, pp. 1414-1419.

[50] J. Aghaei, N. Amjady, A. Baharvandi, and M.-A. Akbari, "Generation and transmission expansion planning: Milp-based probabilistic model," Power Systems, IEEE Transactions on, vol. 29, no. 4, pp. 1592-1601, July 2014.

[51] S. Asadamongkol and B. Eua-arporn, "Transmission expansion planning with $\{\mathrm{AC}\}$ model based on generalized benders decomposition," International Journal of Electrical Power \& Energy Systems, vol. 47, no. 0 , pp. 402 - 407, 2013. [Online]. Available: http://www. sciencedirect.com/science/article/pii/S0142061512006400

[52] S. Binato, M. Pereira, and S. Granville, "A new benders decomposition approach to solve power transmission network design problems," Power Systems, IEEE Transactions on, vol. 16, no. 2, pp. 235-240, May 2001.

[53] A. Conejo, E. Castillo, R. Minguez, and R. Garcia-Bertrand, Decomposition Techniques in Mathematical Programming - Engineering and Science Applications, 1st ed. Springer-Verlag, 2006, ISBN 978-3-54027685-2.

[54] H. Ergun, B. Rawn, R. Belmans, and D. Van Hertem, "Technology and topology optimization for multizonal transmission systems," Power Systems, IEEE Transactions on, vol. 29, no. 5, pp. 2469-2477, Sept 2014.

[55] O. Gjerde, L. Warland, L. Aleixo, and I. H. Doskeland, "Integrated approach for reliability of electricity supply analysis studies of demonstration network," Cigre Session, Aug. 2012.

[56] Hakan Ergun, "Grid Planning for the Future Grid, Optimizing Topology and Technology Considering Spatial and Temporal Effects," Ph.D. dissertation, University of Leuven, Faculty of Engineering Science, January 2015.

[57] H. Ergun, D. Van Hertem, and R. Belmans, "Identification of Power Injection Capabilities for Transmission System Investment Optimization,' 10th IEEE PowerTech Conference, June 2013, Grenoble, France.

[58] S. P. Vajjhala and P. S. Fischbeck, "Quantifying siting difficulty: A case study of us transmission line siting," Energy Policy, vol. 35, no. 1, pp. $650-671,2007$

[59] E. Dijkstra, "A Note on Two Problems in Connexion with Graphs," Numerische Mathematik, vol. 1, no. 1, pp. 269-271, 1959.

[60] R. Dechter and J. Pearl, "Generalized Best-first Search Strategies and the Optimality of A*," J. ACM, vol. 32, no. 3, pp. 505-536, Jul. July 1985.

[61] European Climate Foundation (ECF), "Roadmap 2050, A Practical Guide To A Prosperous, Low-Carbon Europe," http://www.roadmap2050. eu/attachments/files/Volume1_fullreport_PressPack.pdf, ECF, Tech. Rep., 2010.

[62] — "Power Perspectives 2030 - On the road to a decarbonised power sector," http://www.roadmap2050.eu/attachments/files/ PowerPerspectives2030_FullReport.pdf, ECF, Tech. Rep., 2011.

[63] Socio-economic Data and Applications Center. (2014) Population density map of Europe. http://sedac.ciesin.columbia.edu/maps/gallery/ search/2?facets=theme:population $\&$ facets=region:europe. 
[64] ـ (2014) Elevation map of Europe. http://sedac.ciesin.columbia.edu/ maps/gallery/search?facets=region\%3Aeurope\&contains=elevation.

[65] Q. Zhou and J. Bialek, "Approximate model of european interconnected system as a benchmark system to study effects of cross-border trades," Power Systems, IEEE Transactions on, vol. 20, no. 2, pp. 782-788, May 2005.

[66] Red Electrica de Espana. (2015) Real-time generation and demand. http: //www.ree.es/en/activities/realtime-demand-and-generation.

[67] Rseau de Transport d'lectricit. (2015) Record of power consumption. http://clients.rte-france.com/lang/an/visiteurs/vie/vie_stats_conso_ inst.jsp.

[68] B. Hayes, I. Ilie, A. Porpodas, S. Djokic, and G. Chicco, "Equivalent Power Curve Model of a Wind Farm Based on Field Measurement Data," IEEE PowerTech Trondheim, 19-23 June 2011.

Hakan Ergun (S'10) received his MSc degree (Dipl.-Ing.) in electrical engineering from the Graz University of Technology, Austria in 2009. He obtained his $\mathrm{PhD}$ in electrical engineering from KU Leuven, Belgium in 2015. Currently he is a post-doctoral researcher at KU Leuven - EnergyVille. His research interests are long term transmission system planning and development for large scale renewable integration. He is an active member of IEEE and and Cigré. He was chair of the PES Student Branch Chapter Leuven between 2012 and 2015.

Barry Rawn (M '10) received the $\mathrm{PhD}$ degree in electrical engineering in 2010 from the University of Toronto, where he also received the BASc and MASc degrees in Engineering Science and Electrical Engineering. His research interests include nonlinear dynamics and sustainable energy infrastructure. He has been a post-doctoral researcher in the ELECTA group at the KU Leuven between 2012 and 2015. Currently, he is a Lecturer in Power Systems in the Institute for Energy Futures at Brunel University London.

Ronnie Belmans (S'77-M'84-SM'89-F'05) received the M.Sc. degree in electrical engineering in 1979 and the Ph.D. degree in 1984, both from the University of Leuven (KU Leuven), Belgium, the Special Doctorate in 1989 and the Habilitierung in 1993, both from the RWTH, Aachen, Germany. Currently, he is a full professor with the KU Leuven, teaching electric power and energy systems. His research interests are within the framework of grids, smart grids, the deployment of all the new grid technologies and their integration with new resources and the demand in electricity. He is also Guest Professor at Imperial College of Science, Medicine and Technology, London, UK. Dr. Belmans is a Fellow of the IEEE and the IET. He is also the CEO of EnergyVille, the Executive Director of the Global Smart Grid Federation and Honorary Chairman of the board of Elia, the Belgian transmission grid operator.

Dirk Van Hertem (S'02, SM'09) was born in 1979, in Neerpelt, Belgium. He graduated as a M.Eng. in 2001 from the KHK, Geel, Belgium and as a M.Sc. in Electrical Engineering from the KU Leuven, Belgium in 2003. In 2009, he has obtained his PhD, also from the KU Leuven. In 2010, Dirk Van Hertem was a member of EPS group at the Royal Institute of Technology, in Stockholm, Sweden where he was the program manager for controllable power systems for the $\mathrm{EKC}^{2}$ competence center at KTH. Since spring 2011 he is back at the University of Leuven where he is an assistant professor in the ELECTA group. His special fields of interest are power system operation and control in systems with FACTS and HVDC and building the transmission system of the future, including offshore grids and the supergrid concept. $\mathrm{He}$ is an active member of both IEEE (PES and IAS) and Cigré. 